\title{
Physician initiative crucial to recruiting organ-tissue donors
}

Technologic advances, especially in the development of immunosuppressants, have made organ and tissue transplantation nonexperimental, viable procedures. The number of organ transplants and tissue transfers continues to grow. In 1988, physicians performed nearly 13,000 organ transplants and hundreds of thousands of tissue transfers, such as corneal transplants and bone grafts. One-year survival rates for heart and liver transplant recipients are now $75 \%$ to $85 \%$, up from $30 \%$ to $50 \%$ in the 1970 s.

Although the number of transplantations has increased, the number of organ donations has not kept up with the demand. On Jan 1, 1990, approximately 20,000 potential recipients were on the waiting list of the United Network for Organ Sharing. (This federal agency coordinates organ donation, distribution, and transplantation.) Yet, only 4000 Americans donate organs annually. That means for every 40 Americans who die, only one donates organs or tissues.

The American Osteopathic Association (AOA) recognizes this serious donor shortage. In 1988, the AOA House of Delegates issued a position paper recognizing the need for anatomical gifts and acknowledging that a huge donor pool remains untapped. The delegates supported programs that would increase public awareness and identify organ donors. They also affirmed their support for a federally supported, central donor-recipient information center to provide fair, ethical organ distribution to the most qualified patients.

At a recent conference, "Medicine for the 21st Century: Organ Transplantation," sponsored in part by Northwestern University, 130 experts in healthcare, communication, economics, medical ethics, law, computers, and government policy making met to discuss this matter. The participants made the following recommendations:

- Persons from all economic and racial backgrounds should be encouraged to participate in organ do- nor programs. Distribution of organ donations should be done on an equitable basis.

- Through education, the public and healthcare profession must be motivated to consider the importance of organ donations in family life and medical practice as they pertain to transplantation and biomedical research.

- Healthcare professionals and family members should respect a person's decision to donate organs and tissues and fulfill the person's wishes in the appropriate manner.

- Regarding third-party payers, the same reimbursement criteria used for other medical technologies should be applied to organ and tissue transplantations.

- To enhance tissue availability and use, a nationwide tissue transplantation network should be established. This network would consolidate data collection and enable the distribution of organs and tissue in the most equitable manner possible. Furthermore, it should improve cooperation among the various organ and tissue donor organizations.

- Through joint public and private efforts, a nationwide bone marrow donation volunteer network-large enough to meet the American people's needs-should be established.

Any effort to increase organ donations requires the critical support of hospital administrators, emergency room personnel, and physicians. This teamwork approach helped to increase the number of one-year donor and tissue referrals by approximately $400 \%$ and $500 \%$, respectively, at a Baltimore community hospital.

Results such as these prove that physicians' commitment to this initiative will relieve thousands of patients' and their families' suffering. Ultimately lives will be saved.

THOMAS WESLEY ALLEN, DO Editor in Chief 



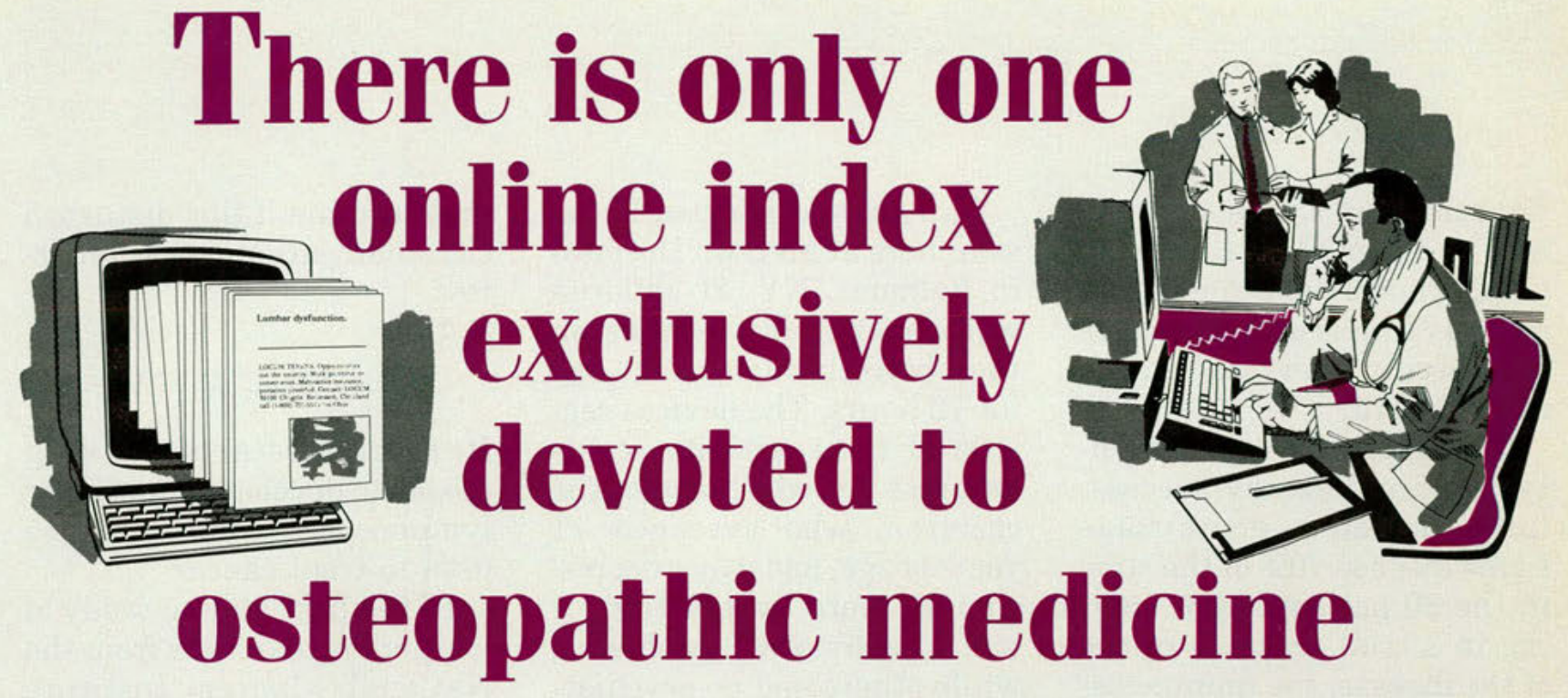

That index is OLIO $+\ldots$. the Osteopathic Literature Index/Online.

OLIO + is unique. $\mathrm{OLIO}+$ is the only bibliographic data base that offers comprehensive coverage of the osteopathic medical literature.

OLIO + subscribers have access to thousands of bibliographic references to articles published since the mid-1970s in major osteopathic journals. Clinical articles, convention reports, legislative and economic information, and news and feature articles can all be located quickly and easily with $\mathrm{OLIO}+$. New records are added every month, and new journal titles are included regularly.

An exclusive feature of $\mathrm{OLIO}+$ is its use of the Glossary of Osteopathic Terminology - no other data base uses this vocabulary for subject access.

$\mathrm{OLIO}+$ can also be searched using the National Library of Medicine's

MeSH headings. All other data elements of OLIO + are also searchable, including personal and institutional names, journal title and year.

OLIO + is easy to use. $\mathrm{OLIO}+$ can be searched by using a

Send registration form to:

American 0steopathic Association OLIO + /Editorial Department 142 E. Ontario St., Chicago, IL 60611 $800 / 621-1773 \times 5865$ or 5895 common command language or a search menu. References contain complete bibliographic information and can be displayed in one of three formats. Searches can be stored for later reference or downloaded to your personal computer.

$\mathrm{OLIO}+$ is inexpensive. If you have access to a modemequipped data terminal or personal computer and telecommunications software, you have all the equipment needed to access $\mathrm{OLIO}+$.

A subscription to $\mathrm{OLIO}+$ is available for $\$ 75$ a year; students, interns and residents pay only $\$ 5$ a year. Subscribers are thereafter billed only for their connect time; there are $n o$ monthly fees or minimum-use fees. The first $\$ 60$ of online time is free.

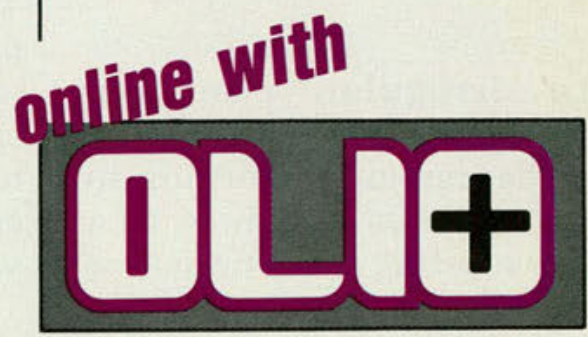



Diagnosing Lyme disease may be easier, thanks to an antibody capture enzyme immunoassay that is more sensitive and specific than standard serologic tests.

The antibody capture enzyme immunoassay successfully detected acute-stage Lyme disease $70 \%$ of the time in the 30 patients participating in a trial. In later stages of the disease, the immunoassay had a $93 \%$ accuracy rate. In this same study, the enzyme-linked immunosorbent assay (ELISA) was accurate $40 \%$ and $70 \%$ of the time, respectively, reported Allen C. Steere, MD, at a recent Lyme disease symposium at Tufts University in Boston.

One of the advantages of this test is that false-positive results are reduced because the assay is followed with an immunoblot test when multiple antibodies are found.

Dr Steere, who is the director of the Lyme Disease Clinic at the New England Medical Center in Boston, believes such stage-specific diagnosis should enhance therapy selection.

An irregular circadian rhythm may be responsible for depression, according to new research presented at a May meeting of the Society of Biological Psychiatry held in New York.
In a study conducted by researchers at McLean Hospital in Belmont, NY, 41 children wore monitoring devices on their belts throughout the day for 72 hours. The devices registered their activity levels and rest periods. Some of the children, who averaged 11 years of age, had major depression, or were hyperactive, or had behavioral problems, while others had no psychiatric illnesses.

Distinct activity patterns were noted between normal volunteers and those with psychiatric or behavioral disorders. Children in the first group had regular circadian cycles, with high activity levels and periods of quiet time during the day and very restful periods at night.

Conversely, seriously depressed children had many more frequent active periods interspersed with quiet periods throughout the day. Their activity patterns seemed related to a marked hemicircadian rhythm cycle.

"I hesitate to say it, because it sounds like science fiction, but you can look at the activity chart of a child and say 'that's hyperactivity...that's depression...that's normal.' It's almost like reading an EKG," says Martin $\mathrm{H}$. Teicher, $\mathrm{PhD}$, one of the McLean researchers and a psychiatrist at Harvard Medical School.

Next, researchers plan to study such patients' families to determine if this disturbed circadian pacemaker is inherited.

Researchers are one step closer to developing "designer lymphocytes" that could be used to treat cancer.

In the first human study of its kind, researchers from the National Cancer Institute have injected a foreign gene into tumor-infiltrating lymphocytes (TIL) taken from patients with advanced melanoma. These TIL cells, which were treated with interleukin2 , were then reinjected into the patients. The gene-altered TIL cells are being used to trace the length of time the treated TIL cells remain in the patients' ciruclatory system and in their tumors.

The treated TIL cells remained in the patients' blood stream and tumors for 189 days and 64 days, respectively. As of late May, tumors had shrunk in two of the five patients in the terminal stage of cancer undergoing this treatment. One woman with skin and pulmonary cancer had no signs of tumors for 10 months. The two remaining patients had yet to be fully assessed at the time these preliminary results were presented at a meeting of the American Society of Clinical Oncology in Washington.

By injecting different genes (continued on page 592) 


\section{EFFICACY...SAFETY...EXPERIENCE}

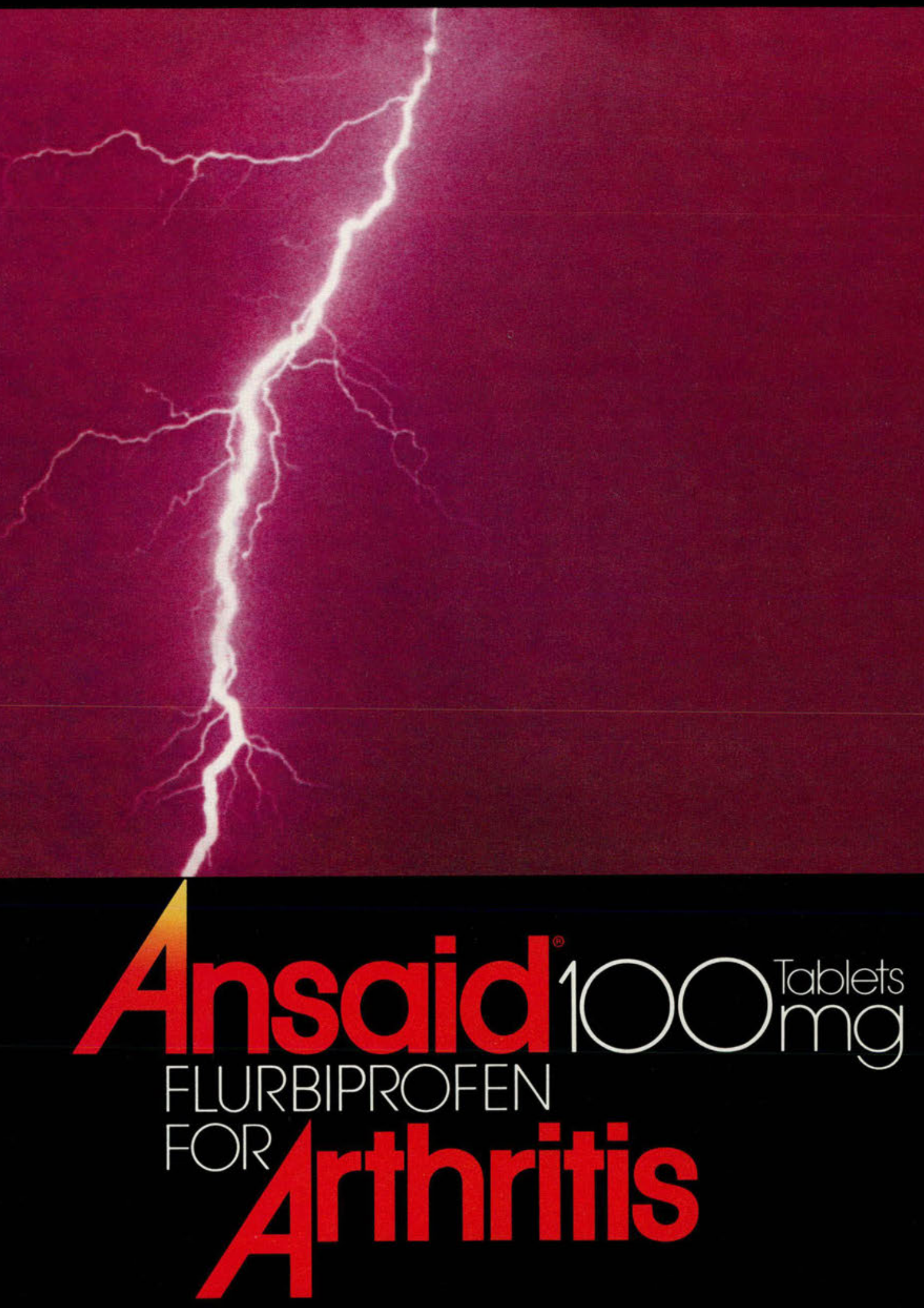

\section{Upjohn}




\section{Arthritis}

INDICATIONS: Acute and long term treatment of signs and symptoms of rheumatoid arthritis and osteoarthritis.

CONTRAINDICATIONS: Hypersensitivity to ANSAID, or if aspirin or any other nonsteroidal antiinflammatory agent induces asthma, urticaria or other allergic type reactions. Fatal asthmatic reactions have been reported in such patients.

WARNINGS: Gastrointestinal effects: Risk of Gl ulcerations, bleeding and perforation with nonsteroidal anti-inflammatory therapy: Serious Gl toxicity can occur at any time, with or without warning symptoms, during chronic treatment. The occurrence is about $1 \%$ after 3-6 months, 2-4\% after a yea.. Patients should be informed of signs and symptoms of serious Gl toxicity and what to do if it occurs. No subset of patients not at risk has been identified. Prior history of serious $\mathrm{Gl}$ events and other risk factors of peptic ulcer disease, e.g., alcoholism, smoking, etc., have been associated with increased risk. The elderly and debilitated tolerate ulceration and bleeding less well. Higher doses probably carry a greater risk Gl ulceration and bleeding can occur without warning symptoms and chronically treated patients should be followed

PRECAUTIONS: Patients with impaired renal or hepatic function: Use ANSAID and similar agents cautiously. Pharmacokinetics have not been studied in patients with decreased liver function.

Renal Effects: Rats develop renal papillary necrosis at dosages equivalent to human therapeutic levels, as do monkeys given 20-40 times the human dose. In clinical studies of ANSAID, kidney function tests were done monthly and renal effects were similar to those seen with other nonsteroidal anti-inflammatory drugs. A second form of renal toxicity has been seen in patients with prerenal conditions that reduce rena blood flow or blood volume. A nonsteroidal anti-inflammatory drug may cause dose-dependent reduction in prostaglandin formation and precipitate overt renal decompensation. Patients at greatest risk are those with impaired renal or hepatic function, heart failure, those taking diuretics or the elderly. Drug discontinuation usually leads to recovery. Patients at high risk on chronic treatment should have rena function monitored if they have signs or symptoms that may be consistent with mild azotemia, e.g. malaise fatigue loss of appetite Occasionally BUN and serum creatinine may be elevated without signs ar symptoms. Flurbiproten is excreted by the kidneys and pharmacokinetics are changed by renal failus c) ccumulation of flurbiprofen metabolites.

Liver tests: Borderline elevations of liver function tests may occur in up to $15 \%$ of patients, and may progress, remain unchanged or disappear with continued treatment. Patients with signs and/or symptoms or with an abnormal liver function test should be evaluated further.

Anemia: Patients treated long term who have initial hemoglobin values under $10 \mathrm{~g} / \mathrm{dL}$, should have periodic hemoglobin values.

Fluid retention and edema: Fluid retention and edema have been reported so use ANSAID with caution in patients with conditions such as cardiac decompensation or hypertension.

Vision Changes: Blurred and/or diminished vision has been reported. Patients with eye complaints should have periodic ophthalmologic exams.

Effect on platelets and coagulation: Platelet aggregation is inhibited and bleeding time prolonged; patients who may be adversely affected should be carefully observed.

Information for patients: Physicians and patients may wish to discuss potential risks and likely benefits.

Drug Interactions: Anticoagulants: Bleeding parameters are affected, clinical bleeding has been reported. Aspirin: Flurbiprofen levels were $50 \%$ lower. Concurrent use is not recommended. Betaadrenergic Blockers: Pharmacokinetics and heart rate reduction are not affected, hypotensive adrenergic Blockers: increase in area under the flurbiprofen serum concentration curve. Diuretics: Patients receiving increase in area under the flurbiprofen serum concentration curve. Diurefics: Patients
furosemide or thiazides should be closely observed to make sure the desired effect is obtained.

Carcinogenesis, mutagenesis, impairment of fertility: No evidence.

Teratogenic effects: Pregnancy category B: No effect in animals. Not recommended for use in pregnancy.

Labor and delivery, nursing mothers, pediatric use: Use is not recommended

ADVERSE REACTIONS: $9.4 \%$ of 4123 patients dropped out of studies because of an a dr. Incidence >1\%: Gastrointestinal: Dyspepsia*, diarrhea*, abdominal pain*, nausea*, constipation, Gl bleeding. flatulence, elevated liver enzymes and vomiting. Central nervous system: Headache*. "stimulation" (eg. anxiety, insomnia, reflexes increased tremor) and "inhibition" (en amnesia, asthenia, somnolence

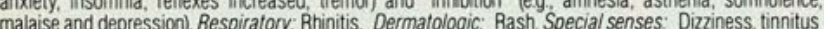
and changes in vision. Genitourinary: Signs and symptoms suggesting a urinary tract infection* Body and changes in vision. Genitourinary: Signs and symptoms suggest

as a whole: Edema* Metabolic,
*Reaction in 3 to $7 \%$ of patients.

Incidence < $1 \%$ (Causal relationship probable): Gastrointestinal: Peptic ulcer disease (See Warnings), gastritis, blody diarthea stomatitis, esophageal disease hematemesis and hepatitis, cholestatic and gastrit, boty iaundice. Central nervous system Ataxia cerebrovascular ischemia, confusion paresthesia and twitching. Hematologic: Decrease in hemoglobin and hematocrit, iron deficiency paresthesia and twitching. Hematologic: Decrease in hemoglobin and hematocrit, iron deficiency anemia, leukopenia, eosinophilia and ecchymosis, thrombocytopenia, hemolytic anemia and aplastic
anemia. (See Precautions) Respiratory: Asthma and epistaxis. Dermatologic: Angioedema, urticaria,
eczema and pruritus; photosensitivity, toxic epidermal necrolysis and exfoliative dermatitis. Special eczema and pruritus; photosensitivity, toxic epidermal necrolysis and exfoliative dermatitis. Special senses: Conjunctivitis and parosmia. Genitourinary: Hematuria and impairment of renal function, interstitial nephritis. Body as a whole: Anaphylactic reactions, chills, tever. Melabolic Nulritionat. Hyperuricemia. Cardiovascular: Heart failure, hypertension, vascular disease and vasodilatation.

Incidence <1\% (Causal relationship unknown): Gastrointestinal: Periodontal abscess, appetite changes, cholecystitis and dry mouth. CNS: Convulsion, meningitis, hypertonia, cerebrovascular accident, emotional lability and subarachnoid hemorrhage. Hematologic: Lymphadenopathy. RespiraIory: Bronchitis, laryngitis, dyspnea, pulmonary embolism, pulmonary infarct, hyperventilation. Dermatologic: Alopecia, nail disorder, herpes, dry skin and sweating. Special senses: Ear disease, corneal opacity, glaucoma, retrobulbar neuritis, change in taste, transient hearing loss, retinal hemorrhage Genitourinary: Menstrual disturbances, vaginal and uterine hemorrhage, vulvovaginitis, prostate disease. Metabolic/nutritional: Hyperkalemia Cardiovascular: Arrthythmias, angina pectoris and myocardial infarction. Musculoskeletal: Myasthenia.

DOSAGE AND ADMINISTRATION: 200 to $300 \mathrm{mg}$ daily, administered bid, tid or qid. (Most experience in rheumatoid arthritis has been with tid or qid dosage. Dose should be tailored to severity of symptoms and patient response.

Store at controlled room temperature $\left(15-30^{\circ} \mathrm{C}\right)$

Federal law prohibits dispensing without a prescription. capable of producing immunoregulatory agents, such as interferon, into TL cells, researchers hope to treat a variety of cancers.

"We can't cure all patients all the time," comments Steven Rosenberg, MD, one of the NCI researchers, "but this treatment is in the infancy of its development. Expectations have to be realistic."

\section{A nonhormonal treatment} for osteoporosis in postmenopausal women increases bone mass and decreases fractures.

In a double-blind study, 66 women with postmenopausal osteoporosis were given oral etidronate $(400 \mathrm{mg} /$ day $)$ or a placebo for 2 weeks, followed by 13 weeks without drugs. All women were given calcium and vitamin D supplements during this study, which lasted for 150 weeks.

Fracture rates differed significantly between the two groups, particularly during the last two thirds of the study. The fracture rate in women receiving etidronate was 6 per 100 patient years, compared with 54 fractures per 100 patient years in the placebo group. By the end of this 3 -year trial, an $8 \%$ increase in bone mass was noted among women in the treatment group, while a $2.7 \%$ decline occurred in women receiving placebo.

(continued on page 594) 


\section{In bronchitis and pneumonia...}
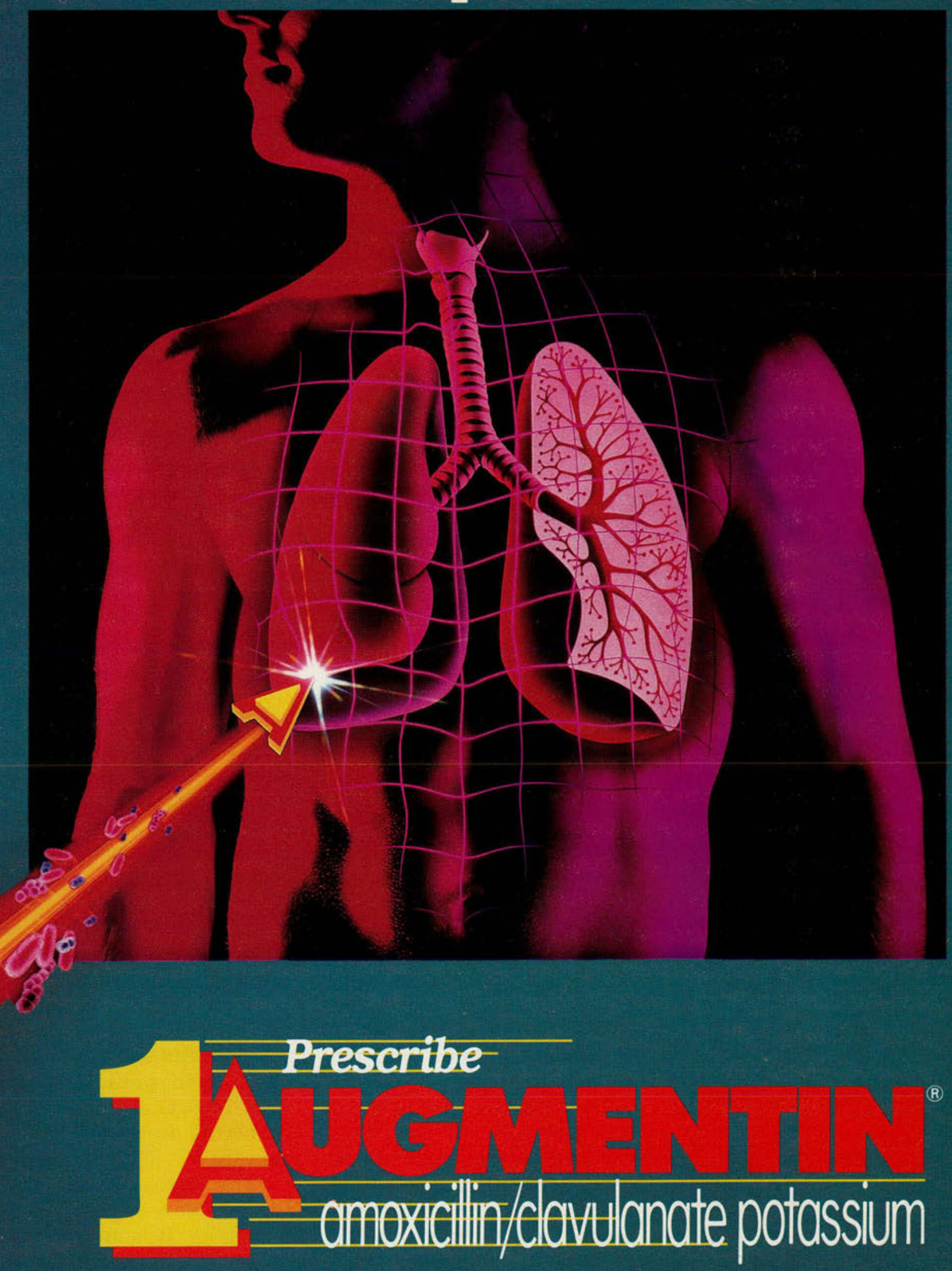

\section{First time, first-line for clinical certainty}


Indications and Usage: AUGMENTIN ${ }^{*}$ is indicated in the treatment of intections caused by susceptible strains of the designated organisms in the conditions Lower Respiratory Infections caused by $\beta$-lactamase-producing strains of Hemophilus influenzae and Branhamella catarrha

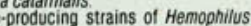
Sinusitis caused by $\beta$-lactamase-producing strains of Hemophilus influenzae and Branhamella catarmalis.

Skin and Skin Structure Infections caused by $\beta$-lactamase-producing strains of Staphylococcus aureus, E. colli, and Klebsiella spo Urinary ractinfections caused by $\beta$-lactamase-producing strains of $E$. colli. While AUGMENTN is indicated only for the conditions listed above. infections caused by ampicillin susceptible organisms are also amenable to AUGMENTIN mpicillin susceptible organisms and $\beta$-lactamase-producing organisms suscepible to AUGMENTIN should not require the addition of another antibiotic. Bacteriological studies, to determine the causative organisms and their suscepti-
bility to AUGMENTIN, should be performed together with any indicated surgical procedures. and susceptibility studies to determine the causative organisms and their susceptibility to AUGMENTIN when there is reason to believe the infection may involve
any of the $\beta$-lactamase-producing organisms listed above. Once the results are
known. therapy should be ad justed if appropriate.

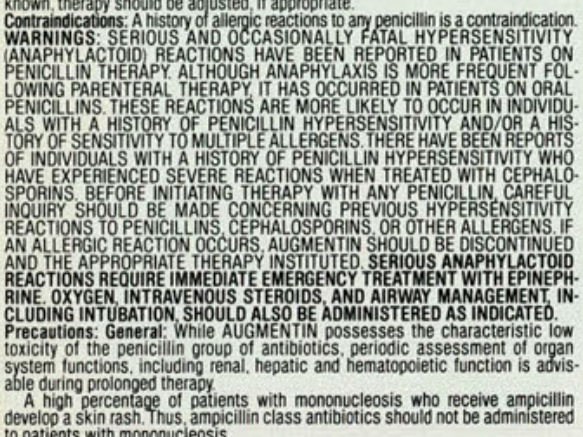
The possibility of superinfections with mycotic or bacterial pathogens should be kept in mind during therapy If superinfections occur (usually involving
Pseudomonas or Candida), the orug should be discontinued and/or appropriate Orug Interactions: Probenecid decreases the renal tubular secretion of amoxicillin. Concurrent use with AUGMENTIN may result in increased and prolonged blood The concurrent administration of allopurinol and ampicillin increases substanvally the incidence of rashes in patients receiving both drugs as compared to patients receiving ampicillin alone. It is not known whether this potentiation of There are no data with AUGMENTIN and allopurinol administered concurrently. AUGMENTiN should not be co-administered with Antabuse
Carcinogenesis. Mutagenesis, Impairment of frertility: Long-term studies animals have not been performed to evaluate carcinogenic or mutagenic potential. Pregnancy (Category B): Reproduction studies have been performed in mice and
ats at doses uo to ten (10) times the human dose and have revealed no evidence of impaired fertility or harm to the fetus due to AUGMENTIN. There are, however no adequate and well-controlled studies in pregnant women. Because animal eproduction studies are not always predictive of human response, this div abor and Delivery. Oral ampicillin class antibiotics are generally poorly absorbed during labor. Studies in guinea pigs have shown that intravenous administration
of ampicillin decreased the uterine tone, frequency of contractions, height of contractions and duration of contractions. Howevet it is not known whether the use of AUGMENTIN in humans during labor or delivery has immediate or delayed adverse effects on the fetus, prolongs the duration of labor or increases the
kilihood that forceps delivery or other obstetrical intervention or resuscitation of Nursing Mothers: Ampicillin class antibiotics are excreted in the milk; therefore. caution should be exercised when AUGMENTIN is administered to a nursing woman. Adverse Reactions: AUGMENTIN is generally well tolerated. The majority of side effects observed in clinical trials were of a mild and transient nature and less
han $3 \%$ of patients discontinued therapy because of drug related side effects han $3 \%$ of patients discontinued therapy because of drug related side effects
The most frequently reported adverse effects were diarrhea/loose stools $(9 \%)$ nausea (3\%), skin rashes and urticaria $(3 \%)$, vomiting (1\%), and vaginitis $(1 \%)$. the higher recommended dose. Other less frequently reported reactions include: boominal discomfort, flatulence and headache

The following adverse reactions have been reported for ampicillin class Gastrointestina: Diarmea, nausea, vomiting, indigestion, gastritis, stomatitis, glossitis. lack "hairy "Tongue, enterocolitis and pseudomembranous colitis. ke reactions (urticaria or skin rash accompanied by arthritis/arthralgia, myalgia and frequently fever), erythema multiforme (frarely Stevens-Johnson Syndrome)

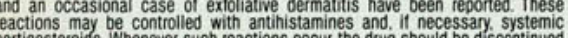
corticosteroids. Whenever such reactions occut the dirug should be discontinued unless the opinion of the physician dictates otherwise. Serious and occasional

iver: A moderate rise in SGOT and/or SGPT has been noted in patients treated with ampicilin class antibiotics as well as with Auche cephalosporins, transient hepatitis and cholestatic jaundice have been reported

emic and Lymphatic Systems: Anemia, thrombocytopenia, thrombocytopenic herapy with penicillins. These reactions are usually reversible on discontinuation

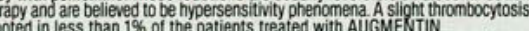
(t)
Central Nervous Srstem. Reversible hyperactivity. agitation, anxiety. insomnia, Confusion behavioral chanoes, and/or dizziness have been reported rarely.
Dosage: Adults: The usual adult dose is one AUGMENTIN " 250 tablet every Dosage: Adults: The usual adult dose is one AUGMENTIN 250 tablet every dOse should be one AUGMENTIN ' 500 ' tablet every eight hours. Since both the AUGMENTIN '250' and ' 500 ' tablets contain the same 250 ' tablets are not equivalent to one AUGMENTIN ' $500^{\prime}$ ' tablet. Therefore two AUGMENTIN ' 250 ' tablets should not be substituted for one AUGMENTIN
500 ' tablet for treatment of more severe infections. 500 ' tablet for treatment of more severe infections.
Children: The usual dose is $20 \mathrm{mg} / \mathrm{kg} /$ day. based on amoxicillin component, in divided doses every eight hours. For otitis media. sinusitis and other more severe intections, the dose should be $40 \mathrm{mg} / \mathrm{kg} /$ day, based on the amoxicillin component. 250 chewable tablets.
Children weighing $40 \mathrm{~kg}$ and more should be dosed according to the adult recommendations,
Likewise, the degree of spinal deformity stabilized after 1 year of etidronate therapy. No significant side effects were noted among women in the treatment group.

"Etidronate cyclical therapy increases trabecular bone mass in the spine without negatively affecting cortical bone found in such areas as the hip and wrist, " explains Tommy Storm, MD, the study's primary investigator.

Etidronate cyclical therapy has not yet been approved by the Food and Drug Administration (FDA) for the treatment of osteoporosis.

Complete results appear in the May 3 issue of The New England Journal of Medicine.

In utero surgical repair of congenital diaphragmatic hernia has been performed successfully on two fetuses.

The May 31 issue of The New England Journal of Medicine reports on the first successful surgery in which a 24.5 week-old male fetus underwent this experimental procedure. Surgeons brought out the left arm of the fetus through an incision in the uterus. The left side of the chest and flank were positoned under the hysterotomy. Surgeons removed the stomach, large and small intestines, and spleen from the chest of the fetus. A Gore-Tex patch closed the defective dia- phragm. Warm Ringer's lactate solution replaced the air in the emptied left-sided chest cavity. Throughout the 54-minute operation, the fetus was monitored via pulse oximetry, electrocardiography, and sonography.

At 32 weeks' gestation, the infant was delivered via cesarean section. He was gradually weaned from a respirator over a 1-month period, but required afundoplication for persistent gastroesophageal reflux. As of late May, the infant remains healthy.

Michael R. Harrison, MD, developed this procedure and has performed eight operations since 1984, six of which proved unsuccessful. Uterine irritability following hysterotomy is only one of the unresolved problems with this procedure.

The second successful procedure was performed on a female fetus on March 3. By the second day of life, the baby girl was breathing without respiratory assistance and is reportedly doing fine at press time.

Approximately $75 \%$ of fetuses with prenatally confirmed congenital diaphragmatic hernia die despite postnatal care.

"The disease he is attacking is a very big problem in pediatrics. It is an important approach that ought to continue to be investigated," commments Joseph Vacanti, MD, of Children's Hospital in Boston. 


\section{In hypertension, \\ There's safety in these numbers.}

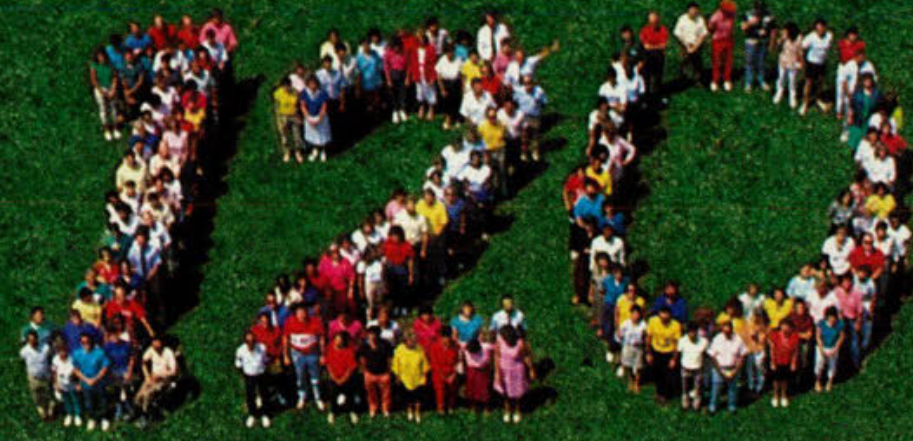

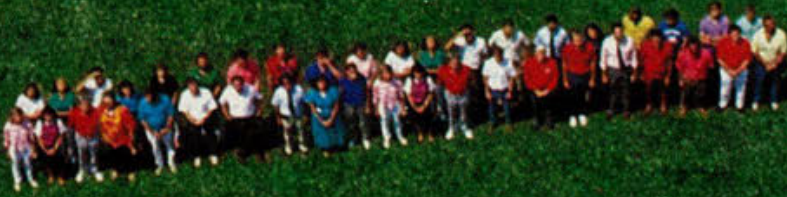
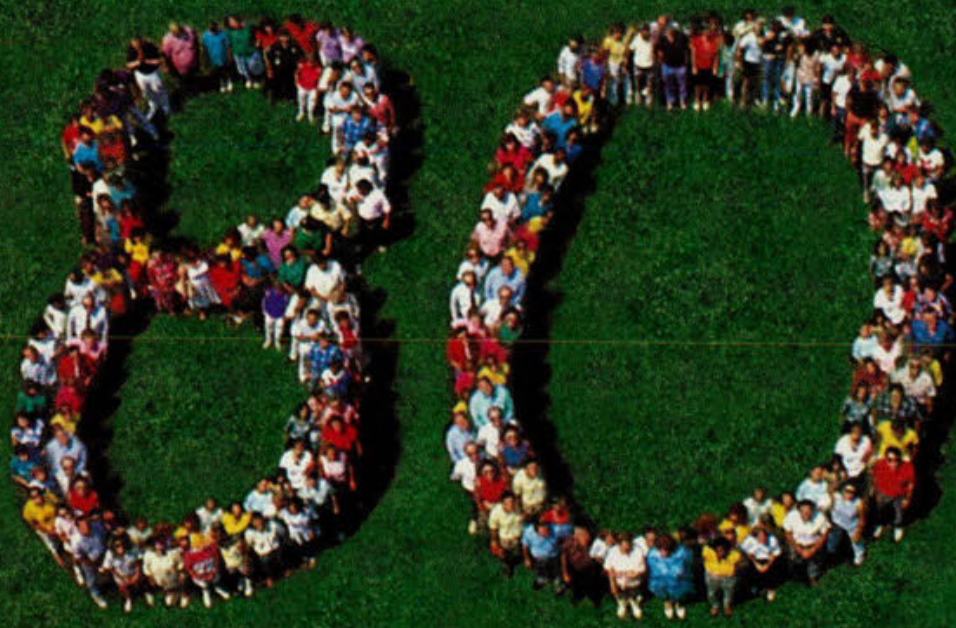

\section{MINIPRESS (prazosin $\mathrm{HCl}$ ) \\ Blood pressure control that leaves other CHD risk factors unaffected}

1. Effectively reduces high blood pressure

2. Does not adversely affect the lipid profile ${ }^{26}$

3. Does not impair exercise capacity'

4. Has no significant effect on glucose metabolism ${ }^{7.8}$

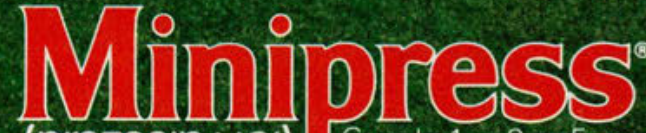

(prazOSIHHCl) $\sqrt{ }$ capsules $1 \mathrm{mg}^{2 \mathrm{mg} 5 \mathrm{mg}}$

For Initial 2heraipy of Hypertension

Most common side effects, generally mild and transient, are: dizziness, headache, drowsiness, palpitations, and nausea. Syncope has been reported in about $0.15 \%$ of patients at the recommended initial dose of $1 \mathrm{mg}$. 